\title{
QUASIPOLARITY OF SPECIAL MORITA CONTEXT RINGS
}

\author{
O. GÜRGÜN, S. HALICIOGLU, AND A. HARMANCI
}

Received 03 April, 2017

\begin{abstract}
In this paper, we introduce a new concept of Morita context rings which we call special Morita context rings. We determine the conditions under which this kind of rings are quasipolar. We use special Morita context rings to extend some results of quasipolar rings. Then many of the main results of quasipolar rings are special cases of our results for this general setting. Several basic characterizations and properties of these rings are given.
\end{abstract}

2010 Mathematics Subject Classification: 16S50; 16S70; 16U99

Keywords: quasipolar ring, strongly clean ring, strongly $\pi$-regular ring, Morita context ring, special Morita context ring

\section{INTRODUCTION}

Throughout this paper all rings are associative with identity and modules are unital. The commutant and second commutant of $a \in R$ are defined by $\operatorname{comm}(a)=\{x \in$ $R \mid x a=a x\}, \operatorname{comm}^{2}(a)=\{x \in R \mid x y=y x$ for all $y \in \operatorname{comm}(a)\}$, respectively. $U(R), J(R)$ and $\operatorname{Nil}(R)$ will denote the set of all invertible elements, the Jacobson radical of $R$ and the set of all nilpotent elements, respectively. An element $a$ in a ring $R$ is called quasinilpotent if $1-a x \in U(R)$ for any $x \in \operatorname{comm}(a)$. The set of all quasinilpotent elements of $R$ will be denoted by $Q N(R)$ ([9]). Set $J^{\#}(R)=$ $\left\{x \in R \mid \exists n \in \mathbb{N}\right.$ such that $\left.x^{n} \in J(R)\right\}$. Obviously, $J(R) \subseteq J^{\#}(R) \subseteq Q N(R)$ and $\operatorname{Nil}(R) \subseteq J^{\#}(R) \subseteq Q N(R)$.

The notion of a quasipolar ring was introduced by Harte in his 1991 study on quasinilpotent in rings. An element $a \in R$ is called quasipolar provided that there exists an idempotent $p \in R$ such that $p \in \operatorname{comm}^{2}(a), a+p \in U(R)$ and $a p \in Q N(R)$. A ring $R$ is quasipolar in case every element in $\mathrm{R}$ is quasipolar. Any idempotent $p$ satisfying the above conditions is called a spectral idempotent of $a$. Koliha [11] introduced the concept of a generalized Drazin inverse in a complex Banach algebra. An element $a$ of $R$ is generalized Drazin invertible [12] in case there is an element $b \in R$ satisfying $a b^{2}=b, b \in \operatorname{comm}^{2}(a)$ and $a^{2} b-a \in Q N(R)$. Such $b$, if it exists, is unique; it is called a generalized Drazin inverse of $a$ and will be denoted by $a^{g D}$. Koliha and Patricio [12] proved any quasipolar element $a \in R$ has a unique spectral 
idempotent denoted by $a^{\pi}$, and $a$ is quasipolar if and only if $a$ is generalized Drazin invertible. Quasipolar rings have been studied by several authors [2, 4, 7, 12, 22].

Recall some definitions. A ring is called strongly clean if every element is the sum of an idempotent and a unit which commute (see [16]). Following [1], an element $a \in R$ is said to be strongly $\pi$-regular if $a^{n} \in a^{n+1} R \cap R a^{n+1}$ for some $n \in \mathbb{N}$. An element $a \in R$ is called pseudopolar if there exists $p^{2}=p \in \operatorname{comm}^{2}(a)$ such that $a+$ $p \in U(R)$ and $a p \in J^{\#}(R)$ ([21]). An element $a$ of $R$ is (pseudo) Drazin invertible ([21]) [5] in case there is an element $b \in R$ satisfying $a b^{2}=b, b \in \operatorname{comm}^{2}(a)$ and $\left(a^{2} b-a \in J^{\#}(R)\right) a^{2} b-a \in \operatorname{Nil}(R)$. Such $b$, if it exists, is unique; it is called a (pseudo) Drazin inverse of $a$ and will be denoted by ( $\left.a^{p D}\right) a^{D}$. In 1958, Drazin showed that $a$ is strongly $\pi$-regular if and only if $a$ has a Drazin inverse. Wang and Chen proved that $a$ is pseudopolar if and only if $a$ is pseudo Drazin invertible. By definitions, we conclude that any strongly $\pi$-regular element is pseudopolar, any pseudopolar element is quasipolar and any quasipolar element is strongly clean.

Morita contexts appeared as a key ingredient in the work of Morita that described equivalences between full categories of modules over rings with identities. Morita context rings form a very large class of rings generalizing matrix rings. One of the fundamental results in this direction says that the categories of left modules over the rings $A$ and $B$ are equivalent if and only if there exists a strict Morita context connecting $A$ and $B$. Other applications, though not stated in an explicit form, can be found in various places. A Morita context $(A, B, M, N, \psi, \phi)$ consists of two rings $A$ and $B$, two bimodules ${ }_{A} M_{B},{ }_{B} N_{A}$ and a pair of bimodule homomorphisms

$$
\psi: M \bigotimes_{B} N \rightarrow A \text { and } \phi: N \bigotimes_{A} M \rightarrow B
$$

which satisfy the following associativity:

$$
\psi(m \otimes n) m^{\prime}=m \phi\left(n \otimes m^{\prime}\right) \text { and } \phi(n \otimes m) n^{\prime}=n \psi\left(m \otimes n^{\prime}\right)
$$

for any $n, n^{\prime} \in N, m, m^{\prime} \in M$. These conditions insure that the set of generalized matrices $\left[\begin{array}{cc}a & m \\ n & b\end{array}\right] ; a \in A, b \in B, m \in M, n \in N$ will form a ring, called the ring of the Morita context. A Morita context $\left[\begin{array}{cc}A & M \\ N & B\end{array}\right]$ with $A=B=R, M=N$, and $\psi=\phi=\varphi$ is called a special Morita context. Throughout $\delta$ denotes the ring of a special Morita context $[R, M, \varphi]$.

The main purpose of this paper is to study quasipolarity of special Morita contexts which are a natural generalization of (generalized) full matrix rings $\left(K_{S}(R)\right.$ or $\left.M_{2}(R ; s)\right) M_{2}(R)$. One of the motivations to study the concept of special Morita context rings is to construct nontrivial examples for quasipolar rings (see Examples 1, $2,3)$. In the present paper we use special Morita context rings to extend some results of quasipolar rings (e.g. [2, Theorem 2.10], [10, Theorem 22], [19, Theorem 15, Theorem 18 and Theorem 22] and [21, Theorem 1.4]). 
This paper is organized as follows. In Section 2, the forms of the Jacobson radical and the center of special Morita contexts are determined and some properties of this class of rings are investigated. Furthermore, we define (uniquely) weakly quasipolar rings and determine the relation between quasipolar rings and pseudopolar rings (or equivalently, uniquely strongly $\pi$-rad clean rings). In particular, we show that $R$ is quasipolar if and only if $R$ is uniquely weakly quasipolar (Theorem 2). This gives an affirmative answer to the question in [12, Remark 4.8] and [11, Lemma 2.4]. In Section 3, criteria are obtained for a single element of $\delta$ to be quasipolar for a local ring $R$. As a result, we see that (in Proposition 5) $\delta$ is quasipolar if and only if $\delta$ is weakly quasipolar where $M$ is uniquely bleached and $R$ is local. In Section 4, we determine when a special Morita contexts over a commutative local ring is quasipolar. It is shown that $\delta$ is quasipolar if and only if $x^{2}-x+w=0$ is solvable for every $w \in \operatorname{Im} \varphi$ where $R$ is commutative local and $\operatorname{Im} \varphi \subseteq J(R)$. This extend and improve many known results such as [10, Corollaries 11 and 12] and [19, Theorem 18]. In particular, we prove that if $R$ is commutative local and $\operatorname{Im} \varphi$ is nilpotent, then $\delta$ is quasipolar. This yields the main result of [19] (see Example 1). Several equivalent conditions on quasipolar special Morita context rings over a (commutative) local ring are obtained.

In this paper, the ring of integers modulo $n$ is denoted by $\mathbb{Z}_{n}$, and we write $M_{n}(R)$ and $C(R)$ for the rings of all $n \times n$ matrices over the ring $R$ and the set of all central elements of $R$, respectively. For elements $a, b$ in a ring $R$, we use the notation $a \sim$ $b$ to mean that $a$ is similar to $b$, that is, $b=u^{-1} a u$ for some $u \in U(R)$. We set $(M: J(R))=\{m \in M \mid \varphi(m \bigotimes n) \in J(R)$ for all $n \in M\}$ and $(J(R): M)=\{m \in$ $M \mid \varphi(n \bigotimes m) \in J(R)$ for all $n \in M\}$.

\section{Preliminary RESUlts}

In this section, we decide the forms of the Jacobson radical and the center of special Morita contexts. We introduce (uniquely) weakly quasipolar rings and investigate relations between quasipolar rings and pseudopolar rings (or equivalently, uniquely strongly $\pi$-rad clean rings). We prove that $R$ is quasipolar if and only if $R$ is uniquely weakly quasipolar (Theorem 2). As a result we obtain an affirmative answer to the question in [12, Remark 4.8] and [11, Lemma 2.4].

The Jacobson radical formula of a Morita context was determined by Sands in his 1973 study on Radicals and Morita contexts (see [17]):

Theorem 1. Let $R$ be a ring. Then

$$
J(\delta)=\left[\begin{array}{cc}
J(R) & (M: J(R)) \\
(M: J(R)) & J(R)
\end{array}\right]=\left[\begin{array}{cc}
J(R) & (J(R): M) \\
(J(R): M) & J(R)
\end{array}\right] .
$$

Corollary 1. Let $R$ be a ring and $\operatorname{Im} \varphi \subseteq J(R)$. Then

$$
J(\delta)=\left[\begin{array}{cc}
J(R) & M \\
M & J(R)
\end{array}\right] .
$$


Proposition 1. Let $R$ be a ring and $\operatorname{Im} \varphi \subseteq J(R)$. If $J(R)$ is nilpotent, then $J(8)$ is nilpotent.

Proof. Assume that $[J(R)]^{t}=0$ for some $t \in \mathbb{N}$. By direct calculation one sees that

$$
[J(8)]^{t} \subseteq\left[\begin{array}{cc}
{[J(R)]^{t}+\operatorname{Im} \varphi} & M \\
M & {[J(R)]^{t}+\operatorname{Im} \varphi}
\end{array}\right] \subseteq\left[\begin{array}{cc}
\operatorname{Im} \varphi & M \\
M & \operatorname{Im} \varphi
\end{array}\right]=: T .
$$

Further, we have

$$
T^{2}=\left[\begin{array}{cc}
\operatorname{Im} \varphi & \operatorname{Im} \varphi \cdot M \\
\operatorname{Im} \varphi \cdot M & \operatorname{Im} \varphi
\end{array}\right] \text {, and so } T^{2 t}=\left[\begin{array}{cc}
{[\operatorname{Im} \varphi]^{t}} & {[\operatorname{Im} \varphi]^{t} \cdot M} \\
{[\operatorname{Im} \varphi]^{t} \cdot M} & {[\operatorname{Im} \varphi]^{t}}
\end{array}\right]=0 .
$$

Therefore $[J(\&)]^{2 t^{2}}=0$, that is, $J(\&)$ is nilpotent, as desired.

The following lemma is verified by direct calculation.

Lemma 1. Let $R$ be a ring. Then

$$
C(\&)=\left\{\left[\begin{array}{ll}
a & 0 \\
0 & b
\end{array}\right] \mid a, b \in C(R) \text { and } a m=m b, b m=\text { ma for all } m \in M\right\} .
$$

Lemma 2. Let $R$ be a ring and $\operatorname{Im} \varphi \subseteq J(R)$. Then $\left[\begin{array}{cc}u & m \\ n & v\end{array}\right]$ is invertible in 8 if and only if $u, v \in U(R)$.

Proof. Since units always lift modulo the Jacobson radical, it is clear by Corollary 1.

Lemma 3. Let $R$ be a local ring and let $\alpha^{2}=\alpha \in \delta$. Then there exist $\beta, \gamma \in U(8)$ such that $\beta \alpha \gamma=\left[\begin{array}{ll}* & 0 \\ 0 & *\end{array}\right]$.

Proof. Write $\alpha=\left[\begin{array}{cc}e & m \\ n & f\end{array}\right]$ where $e, f \in R$ and $m, n \in M$. Since $\alpha^{2}=\alpha$, we have

$$
e=e^{2}+\varphi(m \otimes n), m=e m+m f, n=n e+f n, f=f^{2}+\varphi(n \otimes m) .
$$

If $e \in U(R)$, then we see that

$$
\left[\begin{array}{cc}
1 & 0 \\
-n e^{-1} & 1
\end{array}\right]\left[\begin{array}{cc}
e & m \\
n & f
\end{array}\right]\left[\begin{array}{cc}
1 & -e^{-1} m \\
0 & 1
\end{array}\right]=\left[\begin{array}{cc}
e & 0 \\
0 & f-\varphi\left(n e^{-1} \otimes m\right)
\end{array}\right]
$$

Similarly, if $f \in U(R)$, then there exist $\beta, \gamma \in U(8)$ such that $\beta \alpha \gamma=\left[\begin{array}{ll}* & 0 \\ 0 & *\end{array}\right]$. Now assume that $e, f \in J(R)$. Then $\alpha \in J(8)$, and so it is zero. We complete the proof. 
In view of [18, Theorem 4], we have the following result.

Corollary 2. Let $R$ be a local ring and let $\alpha^{2}=\alpha \in 8$. Then there exists $a$ $\beta \in U(\delta)$ such that $\beta \alpha \beta^{-1}=\left[\begin{array}{ll}* & 0 \\ 0 & *\end{array}\right]$.

Let $M$ be an $R$ - $R$-bimodule and let $a \in R . l_{a}: M \rightarrow M$ and $r_{a}: M \rightarrow M$ denote, respectively, the abelian group endomorphisms of $M$ given by $l_{a}(m)=a m$ and $r_{a}(m)=m a$ for all $m \in M$.

Lemma 4. Let $\alpha=\left[\begin{array}{ll}x & 0 \\ 0 & y\end{array}\right], X=\left[\begin{array}{cc}a & m \\ n & b\end{array}\right] \in$ 8. Then $X \in \operatorname{comm}(\alpha)$ if and only if $a \in \operatorname{comm}(x), b \in \operatorname{comm}(y), m \in k e r\left(l_{x}-r_{y}\right)$ and $n \in k e r\left(l_{y}-r_{x}\right)$.

Proof. It is straightforward.

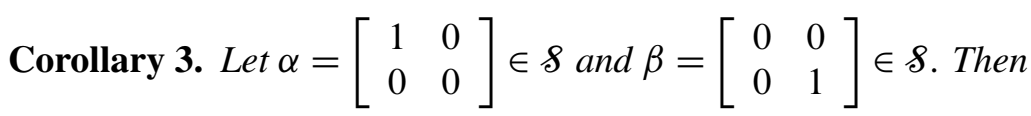

$$
\operatorname{comm}(\alpha)=\left\{\left[\begin{array}{ll}
a & 0 \\
0 & b
\end{array}\right] \mid a, b \in R\right\}=\operatorname{comm}(\beta) \text {. }
$$

Lemma 5. Let $R$ be a ring. Then $\left[\begin{array}{cc}a & m \\ n & b\end{array}\right] \mapsto\left[\begin{array}{cc}b & n \\ m & a\end{array}\right]$ is an automorphism of 8.

Remark 1. If $R$ is isomorphic to a ring $S$ by $f$, then $a \in R$ is quasipolar if and only if $f(a)$ is quasipolar in $S$.

Lemma 6. Let $R$ be a local ring. Then $\left[\begin{array}{ll}a & 0 \\ 0 & b\end{array}\right] \in Q N(\&)$ if and only if $a, b \in$ $J(R)$ if and only if $\left[\begin{array}{ll}a & 0 \\ 0 & b\end{array}\right] \in J(8)$.

Proof. If $R$ is a local ring, then $J(R)=Q N(R)$, and so the proof is clear.

Wang and Chen prove that ([21, Theorem 1.4]) if there exists $p^{2}=p \in \operatorname{comm}(a)$ such that $a+p \in U(R)$ and $a^{k} p \in J(R)$ for some $k \in \mathbb{N}$, then $p$ is unique if and only if $p \in \operatorname{comm}^{2}(a)$. We extend this result as the following.

Theorem 2. Let $R$ be a ring and assume that $p^{2}=p \in \operatorname{comm}(a), a+p \in U(R)$ and ap $\in R^{q \text { nil }}$ for some $a, p \in R$. Then $p \in \operatorname{comm}^{2}(a)$ if and only if $p$ is unique.

Proof. If $p \in \operatorname{comm}^{2}(a)$, then $p$ is unique by [12, Proposition 2.3]. For the converse, suppose $p$ is unique and let $x a=a x$ for some $x \in R$. Set $c:=x p-p x p$, $b=(a+p)^{-1}(1-p)$. Then $a b^{2}=b, 1-p=a b=b a, p+c=: e$ is an idempotent, $e p=e$ and $p e=p$. We show that $a+e \in U(R)$ and $a e \in Q N(R)$. Since $(a+e)(b+p)=1+a p+p x(1-p)(b+p)=1+p a+p x b=1+p(a+x b)$ is 
invertible if and only if $1+(a+x b) p=1+a p$ is invertible, and $a p \in Q N(R), a+e$ is a right invertible element. Further, as $(b+p)(a+e)=1+a p+p x-p x p=1+$ $a p+$ pxab $=1+a p(1+x b)$ is invertible if and only if $1+(1+x b) a p=1+a p$ is invertible, $a+e$ is a left invertible element. Hence $a+e$ is invertible. Let yae $=a e y$ for some $y \in R$. We prove that $1+$ aey $\in U(R)$. We know that $1+$ aey $\in U(R)$ if and only if $1+$ aye $=1+$ ayep $\in U(R)$ if and only if $1+$ paye $=1+$ apye $\in U(R)$ because $e p=e$. Furthermore, pyeap $=$ pyepa $=$ pyea $=$ pyae and appye $=$ apye $=$ apeye $=$ paeye $=$ pyaee $=$ pyae , we conclude that $1+$ apye $\in U(R)$ as $p a \in Q N(R)$. Thus $e=p+c=p$, and so $c=0$ because $p$ is unique. That is, we have $x p=p x p$. Analogously, it can be shown that $p x=p x p$. Therefore $x p=p x$, and so $p \in \operatorname{comm}^{2}(a)$. The proof is completed.

Definition 1. Let $R$ be a ring. Then $a \in R$ is called weakly quasipolar providing that there exists an idempotent $e \in \operatorname{comm}(a)$ such that $a+e \in U(R)$ and $a e \in$ $Q N(R)$. If this representation is unique, then $a \in R$ is called uniquely weakly quasipolar. A ring $R$ is called (uniquely) weakly quasipolar if any element of $R$ is (uniquely) weakly quasipolar.

Remark 2. Lemma 2.4 in [11], Koliha proved that every weakly quasipolar element is quasipolar in a Banach algebra. But it is not true in a ring. Let $R=\mathbb{Z}_{2}\left[t_{1}, t_{2}, \ldots\right]_{\left(t_{1}\right)}$ be a ring of polynomials in countably many indeterminates, localized at the prime ideal $\left(t_{1}\right)$ (for details see [5, Example 2.4.3]). Let $\sigma$ be the map which satisfies $\sigma\left(t_{i}\right)=t_{i}+1$. Then $R[[x ; \sigma]]$ is the skew formal power series local ring over $R$. Consider $A=\left[\begin{array}{cc}t_{2} & 0 \\ 0 & t_{1}\end{array}\right] \in M_{2}(R[[x ; \sigma]])$ where $t_{1} \in J(R[[x ; \sigma]]), t_{2} \in U(R[[x ; \sigma]])$. We directly see that $A$ is weakly quasipolar with $E_{1}=\left[\begin{array}{ll}0 & x \\ 0 & 1\end{array}\right], E_{2}=\left[\begin{array}{ll}0 & 0 \\ 0 & 1\end{array}\right]$. Therefore $A$ is not quasipolar.

By Theorem 2, we have the following result.

Corollary 4. Let $R$ be a ring. The following are equivalent for $a \in R$.

(1) $a$ is uniquely weakly quasipolar.

(2) $a$ is quasipolar.

\section{NONCOMMUtATIVE CASES}

The goal of this section is to investigate quasipolarity of special Morita contexts over local rings. We begin with the following definition.

Definition 2. We say that a bimodule ${ }_{R} M_{R}$ is called (bleached) cobleached provided that for any $a \in J(R), b \in U(R)$, both $l_{a}-r_{b}$ and $l_{b}-r_{a}$ are (surjective) injective on $M$. Further, a bimodule ${ }_{R} M_{R}$ will be called uniquely bleached if, for every $j \in J(R)$ and $u \in U(R), l_{a}-r_{b}$ and $l_{b}-r_{a}$ are surjective as well as injective. 
Huang, Tang and Zhou [10, Corollary 6] proved that if $K_{S}(R)$ is quasipolar, then $R$ is cobleached where $R$ is local. Our next result shows that the latter assumption is superfluous.

Theorem 3. Let $R$ be a ring. If $\delta$ is quasipolar, then $M$ is cobleached.

Proof. Let $u \in U(R), j \in J(R)$, and let $\left(l_{u}-r_{j}\right)(m)=0$ where $m \in M$. Consider $\alpha=\left[\begin{array}{ll}u & 0 \\ 0 & j\end{array}\right] \in$ s. Take $E=\left[\begin{array}{ll}0 & 0 \\ 0 & 1\end{array}\right]$. Then $E \alpha=\alpha E, \alpha+E$ is invertible and $\alpha E$ is quasinilpotent. Since $\alpha$ is quasipolar, by Theorem 2, $E$ must be unique and $E \in \operatorname{comm}^{2}(\alpha)$. Note that $\left[\begin{array}{cc}0 & m \\ 0 & 0\end{array}\right] \in \operatorname{comm}(\alpha)$. So $E$ commutes with $\left[\begin{array}{cc}0 & m \\ 0 & 0\end{array}\right]$, which implies $m=0$. As required.

Proposition 2. Let $R$ be a local ring. Then $\alpha \in S$ is strongly clean if and only if either $\alpha \in U(\&)$, or $I_{2}-\alpha \in U(\&)$, or $\alpha$ is similar to $\left[\begin{array}{ll}a & 0 \\ 0 & b\end{array}\right]$.

Proof. " $\Rightarrow$ " Assume that $\alpha=\left[\begin{array}{ll}x & y \\ z & t\end{array}\right]$ is strongly clean. Then there exists an idempotent $E \in \delta$ such that $\alpha-E \in U(\widehat{\delta})$ and $E \in \operatorname{comm}(\alpha)$. By Corollary 2, there exists $\beta \in U(\delta)$ such that $\beta^{-1} E \beta=\left[\begin{array}{ll}e & 0 \\ 0 & f\end{array}\right]$. Since $R$ is local and $E^{2}=$ $E$, we have $e^{2}=e, f^{2}=f \in\{0,1\}$. If $E=0$, then $\alpha \in U(8)$. If $E=I_{2}$, then $I_{2}-\alpha \in U(\delta)$. It follows that $\alpha$ is similar to $\left[\begin{array}{ll}a & 0 \\ 0 & b\end{array}\right]$ where $a, b \in R$ because $E \in \operatorname{comm}(\alpha)$.

" $\Leftarrow$ " If $\alpha \in U(\&)$ or $I_{2}-\alpha \in U(\&)$, then it is strongly clean. Hence we can assume that $\alpha$ is similar to $\beta=\left[\begin{array}{ll}a & 0 \\ 0 & b\end{array}\right]$. Since $R$ is local, there exist $e^{2}=e, f^{2}=f \in R$ such that $a-e, b-f \in U(R), e \in \operatorname{comm}(a)$ and $f \in \operatorname{comm}(b)$. Therefore $\beta-F \in$ $U(\delta)$ and $\beta F=F \beta$ where $F=\left[\begin{array}{ll}e & 0 \\ 0 & f\end{array}\right]$. That is, $\beta$ is strongly clean. It is well known that if $x$ is strongly clean and $x \sim y$, then $y$ is strongly clean. Thus $\alpha$ is strongly clean, as asserted.

Proposition 3. Let $R$ be a local ring. Then the following statements are equivalent for $\alpha=\left[\begin{array}{ll}x & 0 \\ 0 & y\end{array}\right] \in \delta$.

(1) $\alpha$ is quasipolar.

(2) Both $l_{x}-r_{y}$ and $r_{x}-l_{y}$ are injective.

(3) $\operatorname{comm}(\alpha)=\left\{\left[\begin{array}{ll}a & 0 \\ 0 & b\end{array}\right] \mid a, b \in R\right\}$. 
Proof. (1) $\Rightarrow$ (2) Suppose that $\left(l_{x}-r_{y}\right)(m)=0$ for some $m \in M$ and $\alpha+E \in$ $U(\&)$ where $E=E^{2}=\left[\begin{array}{ll}a & b \\ c & d\end{array}\right] \in \operatorname{comm}^{2}(\alpha)$ and $\alpha E \in Q N(\&)$. By Lemma 4, we get $\left[\begin{array}{ll}1 & 0 \\ 0 & 0\end{array}\right],\left[\begin{array}{cc}0 & m \\ 0 & 0\end{array}\right] \in \operatorname{comm}(\alpha)$. Then $b=0=c, a^{2}=a$ and $d^{2}=d$ because $E^{2}=E \in \operatorname{comm}^{2}(\alpha)$. Since $R$ is local, $a, d \in\{0,1\}$. In view of Corollary 3, $\operatorname{comm}(E)$ is the set of all diagonal matrices in $\delta$. We conclude that $m=0$; that is, $l_{x}-r_{y}$ is injective. Similarly, we see that $r_{x}-l_{y}$ is injective. So holds (2).

(2) $\Rightarrow$ (1) If $x, y \in U(R)$ or $x, y \in J(R)$, then we easily see that $\alpha$ is quasipolar in 8. Let $x \in J(R)$ and $y \in U(R)$. Write $E=\left[\begin{array}{ll}1 & 0 \\ 0 & 0\end{array}\right]$. Then $E^{2}=E \in \operatorname{comm}(\alpha)$ and $\alpha+E \in U(8)$. Let $X=\left[\begin{array}{ll}a & b \\ c & d\end{array}\right] \in \operatorname{comm}(\alpha)$. According to Lemma $4, b \in$ $\operatorname{ker}\left(l_{x}-r_{y}\right)$ and $c \in \operatorname{ker}\left(r_{x}-l_{y}\right)$. By (2), we have $b=0=c$. Hence we get $E \in \operatorname{comm}^{2}(\alpha)$ by Corollary 3. As $\alpha E=\left[\begin{array}{ll}x & 0 \\ 0 & 0\end{array}\right] \in J(\delta)$ by Theorem 1, we have $\alpha E \in Q N(8)$. That is, $\alpha$ is quasipolar in 8 .

(2) $\Rightarrow$ (3) Let $X=\left[\begin{array}{cc}a & m \\ n & b\end{array}\right] \in \operatorname{com} m(\alpha)$. By Lemma 4, we have $m \in \operatorname{ker}\left(l_{x}-\right.$ $\left.r_{y}\right)$ and $n \in \operatorname{ker}\left(r_{x}-l_{y}\right)$. By assumption, we get $m=n=0$, and so $\operatorname{com} m(\alpha)=$ $\left\{\left[\begin{array}{ll}a & 0 \\ 0 & b\end{array}\right] \mid a, b \in R\right\}$.

(3) $\Rightarrow$ (2) Suppose that $\left(l_{x}-r_{y}\right)(n)=x n-n y=0$ for some $n \in M$. This gives that $X=\left[\begin{array}{ll}0 & n \\ 0 & 0\end{array}\right] \in \operatorname{comm}(\alpha)$. By (3), we have $n=0$ and so $l_{x}-r_{y}$ is injective. Similarly, it can be proved that $r_{x}-l_{y}$ is injective. Therefore we complete the proof.

Remark 3. As is well known, if $a b$ is quasipolar and $(a b)^{g D}=c$, then so is $b a$ and $(b a)^{g D}=b c^{2} a$ [15] (or see [6]). In particular, if $a$ is quasipolar and $a^{g D}=c$, then $u^{-1} a u=b$ is quasipolar for any $u \in U(R)$ and $b^{g D}=u^{-1} c u$.

Proposition 4. Let $R$ be a local ring. Then $\alpha \in \delta$ is quasipolar if and only if $\alpha \in U(8)$, or $\alpha \in Q N(8)$, or $\alpha$ is similar to $\left[\begin{array}{ll}a & 0 \\ 0 & b\end{array}\right]$ where $l_{a}-r_{b}, l_{b}-r_{a}$ are injective.

Proof. " $\Rightarrow$ " Assume that $\alpha=\left[\begin{array}{ll}x & y \\ z & t\end{array}\right]$ is quasipolar with spectral idempotent $E=\left[\begin{array}{cc}p & m \\ n & q\end{array}\right]$. Then, by Corollary 2, there exists $\beta \in U(\&)$ such that $\beta^{-1} E \beta=$ $\left[\begin{array}{ll}e & 0 \\ 0 & f\end{array}\right]$. Since $R$ is local and $E^{2}=E$, we have $e^{2}=e, f^{2}=f \in\{0,1\}$. If $E=0$, 
then $\alpha \in U(8)$. If $E=I_{2}$, then $\alpha \in Q N(8)$. It follows that $\alpha$ is similar to $\left[\begin{array}{ll}a & 0 \\ 0 & b\end{array}\right]$ where $a, b \in R$ because $E \in \operatorname{comm}^{2}(\alpha)$. In view of Proposition $3, l_{a}-r_{b}, l_{b}-r_{a}$ are injective, as asserted.

" $\Leftarrow$ " It is clear from Proposition 3 and Remark 3.

Remark 4. Clearly, if $a \in U(R) \cup Q N(R)$, then it is weakly quasipolar. In this case, we say that $a$ is trivial weakly quasipolar. If $a$ is not in $U(R) \cap Q N(R)$, we say that $a$ is non-trivial weakly quasipolar.

Theorem 4. Let $R$ be a local ring. Assume that $\alpha$ is non-trivial weakly quasipolar with $E^{2}=E \in$ \&. The following are equivalent.

(1) $E$ is unique.

(2) $E \in \operatorname{comm}^{2}(\alpha)$.

(3) $\alpha$ is similar to $\left[\begin{array}{ll}a & 0 \\ 0 & b\end{array}\right]$ where $l_{a}-r_{b}, l_{b}-r_{a}$ are injective.

Proof. By Theorem 2, we conclude that (1) $\Leftrightarrow(2)$.

(2) $\Rightarrow$ (3) By assumption, we get $\alpha$ is quasipolar. Then $\alpha$ is similar to $\left[\begin{array}{ll}a & 0 \\ 0 & b\end{array}\right]$ where $l_{a}-r_{b}, l_{b}-r_{a}$ are injective by Proposition 4 .

(3) $\Rightarrow$ (1) In view of Proposition $4, \alpha$ is quasipolar, and so $E$ is unique.

Theorem 5. Let $R$ be a local ring. For any $u \in U(R)$ and $j \in J(R)$, the following are equivalent.

(1) $r_{u}-l_{j}$ is injective and $l_{u}-r_{j}$ is an isomorphism.

(2) For each $m \in M, \alpha=\left[\begin{array}{cc}u & m \\ 0 & j\end{array}\right]$ is quasipolar in $\&$ with $\alpha^{\pi}=\left[\begin{array}{ll}0 & n \\ 0 & 1\end{array}\right]$ for some $n \in M$.

Proof. (1) $\Rightarrow(2)$ Let $m \in M$. Since $l_{u}-r_{j}$ is an isomorphism, there exists $n \in M$ such that $u n-n j=-m$. Write $E=\left[\begin{array}{cc}0 & n \\ 0 & 1\end{array}\right]$. This gives $E \in \operatorname{comm}(\alpha)$. As

$$
\begin{gathered}
(\alpha+E)\left(\left[\begin{array}{cc}
u^{-1} & -u^{-1}(m+n)(j+1)^{-1} \\
0 & (j+1)^{-1}
\end{array}\right]\right) \\
\quad=\left(\left[\begin{array}{cc}
u^{-1} & -u^{-1}(m+n)(j+1)^{-1} \\
0 & (j+1)^{-1}
\end{array}\right]\right),
\end{gathered}
$$

$(\alpha+E)=I_{2}$, we have $\alpha+E \in U(8)$. We show that $E \in \operatorname{comm}^{2}(\alpha)$. Let $X=$ $\left[\begin{array}{ll}x & y \\ z & t\end{array}\right] \in \operatorname{comm}(\alpha)$. This implies that $z=0, t j=j t, u x=x u$ and $u y-y j=$ 
$x m-m t$ because $r_{u}-l_{j}$ is injective. We conclude that

$$
\begin{aligned}
u(y+x n-n t)-(y+x n-n t) j & =u y-y j+u x n-u n t-x n j+n t j \\
& =u y-y j+x u n-u n t-x n j+n j t \\
& =x m-m t+x u n-u n t-x n j+n j t \\
& =x m-m t+x(u n-n j)+(n j-u n) t \\
& =x m-m t+x(-m)+m t \\
& =0 .
\end{aligned}
$$

Therefore $X E=E X$ because $l_{u}-r_{j}$ is injective. Finally, we show that $\alpha E \in$ $Q N(\S)$. Assume $A=\left[\begin{array}{ll}a & b \\ c & d\end{array}\right] \in \operatorname{comm}(\alpha E)$. Then $I_{2}+\alpha E A \in U(\&)$ because $\alpha E=\left[\begin{array}{cc}0 & n j \\ 0 & j\end{array}\right]$, as desired.

(2) $\Rightarrow$ (1) If $m=0$, then $l_{u}-r_{j}, r_{u}-l_{j}$ are injective. It remains to prove that $l_{u}-r_{j}$ is surjective. That is, for each $m \in M$, there exists $n \in M$ such that $u n-n j=m$. Let $m \in M$. We write $\alpha=\left[\begin{array}{cc}u & m \\ 0 & j\end{array}\right]$. By (2), there exists an idempotent $E=\left[\begin{array}{ll}0 & n \\ 0 & 1\end{array}\right]$ such that $E \in \operatorname{comm}^{2}(\alpha), \alpha+E \in U(8)$, and $\alpha E \in Q N(8)$. Since $\alpha E=E \alpha$, we get $u(-n)-(-n) j=m$.

By a similar method of the proof of Theorem 5, we can derive the following.

Theorem 6. Let $R$ be a local ring. For any $u \in U(R)$ and $j \in J(R)$, the following are equivalent.

(1) $l_{u}-r_{j}$ is injective and $r_{u}-l_{j}$ is an isomorphism.

(2) For any $m \in M, \alpha=\left[\begin{array}{ll}u & 0 \\ m & j\end{array}\right]$ is quasipolar in $\&$ with $\alpha^{\pi}=\left[\begin{array}{ll}0 & 0 \\ n & 1\end{array}\right]$ for some $n \in M$.

According to Theorems 5 and 6, the following result is immediate.

Corollary 5. Let $R$ be a local ring. The following statements are equivalent.

(1) $M$ is uniquely bleached.

(2) $\left[\begin{array}{cc}R & M \\ 0 & R\end{array}\right]$ or $\left[\begin{array}{cc}R & 0 \\ M & R\end{array}\right]$ is quasipolar.

Proposition 5. Let $R$ be a local ring and $M$ is cobleached. Then the following are equivalent.

(1) $\alpha \in \mathcal{S}$ is quasipolar.

(2) $\alpha \in \mathcal{S}$ is weakly quasipolar.

Proof. (1) $\Rightarrow(2)$ It is clear. 
(2) $\Rightarrow$ (1) Assume that $E^{2}=E \in \operatorname{comm}(\alpha), \alpha+E \in U(R)$ and $\alpha E \in Q N(8)$. If $E$ is zero or identity in $\delta$, then $\alpha$ is quasipolar. Hence, we deduce that $E$ is similar to the diagonal matrix by Corollary 2. Thus, we have $\alpha$ is similar to the diagonal matrix. Since $M$ is cobleached, we get that $\alpha$ is quasipolar by Theorem 4 .

\section{Commutative cases}

Let $R$ be a commutative ring and $\alpha=\left[\begin{array}{cc}a & m \\ n & b\end{array}\right] \in \mathcal{8}$. In this section, we assume that $\varphi$ satisfying symmetry; that is, $\varphi(m \otimes n)=\varphi(n \otimes m)$ for all $m, n \in M$. We define $\operatorname{det}(\alpha)=a b-\varphi(m \otimes n)$ and $\operatorname{tr}(\alpha)=a+b$, and $r \alpha=\left[\begin{array}{cc}r a & r m \\ r n & r b\end{array}\right]$ for $r \in R$. Note that if $R$ is commutative and $M$ is right $R$-module, then $M$ is left $R$-module where $r m:=m r$ for all $r \in R, m \in M$. Therefore, $M$ is uniquely bleached.

Lemma 7. Let $R$ be a commutative ring and let $\alpha, \beta \in \S$. The following hold:

(1) $\operatorname{det}(\alpha \beta)=\operatorname{det}(\alpha) \operatorname{det}(\beta)$.

(2) $\alpha \in U(8)$ if and only if $\operatorname{det}(\alpha) \in U(R)$.

(3) If $\alpha \sim \beta$, then $\operatorname{det}(\alpha)=\operatorname{det}(\beta)$ and $\operatorname{tr}(\alpha)=\operatorname{tr}(\beta)$.

(4) $\alpha^{2}-\operatorname{tr}(\alpha) \alpha+\operatorname{det}(\alpha) I_{2}=0$.

(5) $\operatorname{det}\left(I_{2}+\alpha\right)=1+\operatorname{tr}(\alpha)+\operatorname{det}(\alpha)$.

Proof. It is straightforward.

Theorem 7. Let $R$ be a commutative ring and let $\alpha \in 8$. Then $\operatorname{det}(\alpha), \operatorname{tr}(\alpha) \in$ $J(R)$ if and only if $\alpha$ is quasinilpotent in 8 .

Proof. Assume that $\operatorname{det}(\alpha), \operatorname{tr}(\alpha) \in J(R)$ and let $\alpha=\left[\begin{array}{cc}a & m \\ n & b\end{array}\right] \in \mathcal{S}$. By Lemma 7(4), we have $\alpha^{2}=\operatorname{tr}(\alpha) \alpha-\operatorname{det}(\alpha) I_{2} \in J(8)$, and so $\alpha \in Q N(8)$. Conversely, suppose that $\alpha \in Q N(S)$. To prove that $\operatorname{det}(\alpha) \in J(R)$, let $y \in R$. It is easy to check that $\beta \alpha=\alpha \beta=\operatorname{det}(\alpha) I_{2}$ where $\beta=\left[\begin{array}{cc}b & -m \\ -n & a\end{array}\right] \in$ s. Since $\alpha \in Q N(8)$, we have that $I_{2}+y \beta \alpha \in U(8)$, and so $1+y \operatorname{det}(\alpha) \in U(R)$. Therefore, we get $\operatorname{det}(\alpha) \in J(R)$. Let $x \in R$. Then we have $I_{2}+x \alpha \in U(8)$, and so $\operatorname{det}\left(I_{2}+x \alpha\right)=1+x \operatorname{tr}(\alpha)+x^{2} \operatorname{det}(\alpha) \in U(R)$ by Lemma 7(2). This gives that $1+x \operatorname{tr}(\alpha) \in U(R)$; thus, $\operatorname{tr}(\alpha) \in J(R)$. The proof is completed.

Corollary 6. Let $R$ be a commutative ring. Then the following statements are equivalent.

(1) $\alpha \in Q N(8)$.

(2) $\operatorname{det}(\alpha), \operatorname{tr}(\alpha) \in J(R)$.

(3) $\alpha^{2} \in J(8)$.

(4) $\alpha^{k} \in J(8)$ for some $k \geq 2$. 
Remark 5. Let $R$ be a commutative ring. Then we conclude that

$$
Q N(8)=J^{\#}(\delta)=\left\{\alpha \in \delta \mid \alpha^{2} \in J(8)\right\} .
$$

Recall that an element $a$ in a ring $R$ is called strongly $\pi$-rad clean if there exists an idempotent $e \in \operatorname{comm}(a)$ such that $a-e \in U(R)$ and $(a e)^{k} \in J(R)$ for some integer $k$.

Corollary 7. Let $R$ be a commutative local ring. The following are equivalent.

(1) $\alpha$ is pseudopolar in 8 .

(2) $\alpha$ is quasipolar in 8 .

(3) $\alpha$ is weakly quasipolar in 8 .

(4) $\alpha$ is strongly $\pi$-rad clean in 8 .

Proof. (1) $\Rightarrow(2),(2) \Rightarrow(3)$ and (4) $\Rightarrow$ (3) are clear. By Remark 5, we deduce that (3) $\Rightarrow$ (4) and (2) $\Rightarrow$ (1). According to Proposition 5, we have (3) $\Rightarrow$ (2).

Theorem 8. Let $R$ be a commutative local ring and let $\alpha \in \mathcal{~}$. Then the following statements are equivalent.

(1) $\alpha$ is quasipolar in 8 .

(2) $\alpha \in U(8)$, or $\alpha \in Q N(8)$, or $x^{2}-\operatorname{tr}(\alpha) x+\operatorname{det}(\alpha)=0$ is solvable.

Proof. (1) $\Rightarrow$ (2) Let $\alpha \in \S$ be quasipolar. We may assume that $\alpha \notin U(8)$ and $\alpha \notin Q N(8)$. Then $\alpha$ is similar to $\beta=\left[\begin{array}{ll}a & 0 \\ 0 & b\end{array}\right]$ where $a \in U(R), b \in J(R)$ by Proposition 4. According to Lemma 7(3), $\operatorname{tr}(\alpha)=\operatorname{tr}(\beta)$ and $\operatorname{det}(\alpha)=\operatorname{det}(\beta)$. This gives $x^{2}-\operatorname{tr}(\alpha) x+\operatorname{det}(\alpha)=x^{2}-\operatorname{tr}(\beta) x+\operatorname{det}(\beta)$. Since $a^{2}-\operatorname{tr}(\beta) a+\operatorname{det}(\beta)=$ 0 , the equation $x^{2}-\operatorname{tr}(\alpha) x+\operatorname{det}(\alpha)=0$ is solvable in $R$.

(2) $\Rightarrow$ (1) If $\alpha$ is invertible or quasinilpotent in $\delta$, then $\alpha$ is quasipolar. Let $\alpha=\left[\begin{array}{ll}a_{11} & a_{12} \\ a_{21} & a_{22}\end{array}\right] \in \delta$ and suppose $x^{2}-\operatorname{tr}(\alpha) x+\operatorname{det}(\alpha)=0$ has roots $a, b \in R$. Since $\alpha \notin U(8) \cap Q N(8)$, we get $\operatorname{det}(\alpha)=a_{11} a_{22}-\varphi\left(a_{12} \otimes a_{21}\right)=a b \in J(R)$ and $\operatorname{tr}(\alpha)=a_{11}+a_{22}=a+b \in U(R)$ by Theorem 7. So one of $a, b$ must be in $U(R)$ and the other must be in $J(R)$. By Lemma 5 and Remark 1, we can assume that $a \in U(R), b \in J(R)$ and $a_{11} \in U(R)$. Let $\beta=\left[\begin{array}{cc}1 & 0 \\ a_{21}\left(a-a_{22}\right)^{-1} & 1\end{array}\right]$. By Lemma 7(2), $\beta \in U(8)$ and easy calculation shows that $\beta^{-1} \alpha \beta$ is an formal triangular matrix in $\left[\begin{array}{cc}R & M \\ 0 & R\end{array}\right]$. Therefore $\alpha$ is quasipolar from Corollary 5 and Remark 3 . We complete the proof.

Now we extend [19, Theorem 15] as follows.

Theorem 9. Let $R$ be a commutative local ring and let $\alpha \in 8$. Then the following statements are equivalent. 
(1) $\alpha$ is non-trivial strongly clean in 8 .

(2) $\operatorname{det}(\alpha) \in J(R), \operatorname{tr}(\alpha) \in 1+J(R)$ and $x^{2}-\operatorname{tr}(\alpha) x+\operatorname{det}(\alpha)=0$ is solvable.

Proof. (1) $\Rightarrow$ (2) It is clear by Proposition 2 and Lemma 7.

$(2) \Rightarrow(1)$ Since $\operatorname{det}(\alpha) \in J(R)$ and $\operatorname{tr}(\alpha) \in 1+J(R)$, we deduce that $\alpha \notin Q N(\delta) \cap$ $U(8)$. By Theorem $8, \alpha$ is quasipolar, and so $\alpha$ is strongly clean.

Remark 6. According to Theorem $9, \alpha \in \mathcal{S}$ is non-trivial strongly clean if and only if it is non-trivial quasipolar in $\delta$ where $R$ is commutative local.

Theorem 10. Let $R$ be a commutative local ring. Then the following are equivalent.

(1) 8 is quasipolar.

(2) For every $\alpha \in \mathcal{S}$ with det $(\alpha) \in J(R)$, one of the following holds:

(i) $\operatorname{tr}(\alpha) \in J(R)$

(ii) $x^{2}-\operatorname{tr}(\alpha) x+\operatorname{det}(\alpha)=0$ is solvable in $R$.

Proof. (1) $\Rightarrow$ (2) Suppose that $\alpha \in \S$ with $\operatorname{det}(\alpha) \in J(R)$. By (1), there exists an idempotent $\alpha \in \delta$ such that $E \in \operatorname{comm}^{2}(\alpha)$ and $\alpha+E \in U(8)$ and $E \alpha \in Q N(8)$. If $E=I_{2}$, then $\alpha \in Q N(8)$ and so $\operatorname{tr}(\alpha) \in J(R)$ by Theorem 7. So we can assume that $\alpha \notin Q N(\delta)$. Since $\operatorname{det}(\alpha) \in J(R), \alpha \notin U(\delta)$ by Lemma 7(2). According to Theorem 8 , the equation $x^{2}-\operatorname{tr}(\alpha) x+\operatorname{det}(\alpha)=0$ is solvable in $R$.

(2) $\Rightarrow(1)$ Let $\alpha \in \delta$. If $\operatorname{det}(\alpha) \in U(R)$, then $\alpha \in U(8)$ and so $\alpha$ is quasipolar. Let $\operatorname{det}(\alpha) \in J(R)$. If $\operatorname{tr}(\alpha) \in J(R)$, then $\alpha$ is quasipolar by Theorem 7. Hence we assume that $\operatorname{tr}(\alpha) \in U(R)$. This gives $\alpha \notin U(8)$ and $\alpha \notin Q N(8)$. By Theorem 8, $\alpha$ is quasipolar and so $\delta$ is quasipolar.

Lemma 8. Let $R$ be a ring and let $u \in U(R) \cap C(R)$. Then $a \in R$ is quasipolar ring if and only if ua is quasipolar in $R$.

Proof. “ $\Rightarrow$ " It follows from [3, Lemma 2].

" $\Leftarrow$ " Assume that $a u=s+q$ where $s$ is strongly regular, $s \in \operatorname{comm}^{2}(a u), q \in$ $Q N(R)$ and $s q=q s=0$ by [7, Corollary 2.17]. Then we get $a=u^{-1} s+u^{-1} q$. It can be shown that $u^{-1} s \in \operatorname{comm}^{2}(a)$ and $u^{-1} s u^{-1} q=u^{-1} q u^{-1} s=0$ because $u \in C(R)$. Since $s$ is strongly regular, there exists $t \in \operatorname{comm}^{2}(s)$ such that $s=s^{2} t$. Multiplying by $u^{-1}$ yields $u^{-1} s=u^{-1} s^{2} t=\left[u^{-1} s\right]^{2} u t$. This gives that $u^{-1} s$ is strongly regular in $R$. To prove $u^{-1} q \in Q N(R)$, let $x u^{-1} q=u^{-1} q x$. As $u$ is central, we deduce that $u^{-1} x q=q u^{-1} x=u^{-1} q x$, and so $1+u^{-1} q x \in U(R)$ because $q \in$ $Q N(R)$, as asserted.

Theorem 11. Let $R$ be a commutative local ring. The following are equivalent.

(1) 8 is pseudopolar.

(2) 8 is quasipolar.

(3) 8 is weakly quasipolar.

(4) 8 is strongly $\pi$-rad clean. 
(5) 8 is strongly clean.

Proof. It suffices to show that (5) $\Rightarrow$ (2). Let $\alpha=\left[\begin{array}{cc}a & m \\ n & b\end{array}\right] \in \delta$. We can further assume that $\alpha \notin U(\delta) \cap Q N(8)$. Then we have $\operatorname{det}(\alpha) \in J(R)$ and $\operatorname{tr}(\alpha) \in U(R)$.

Case I. Let $\varphi(m \otimes n) \in J(R)$. Then we may assume $a \in U(R)$ and $b \in J(R)$ by Lemma 5. We write $a^{-1} \alpha=\left[\begin{array}{ll}1 & p \\ q & j\end{array}\right]$ where $a^{-1} n=q, a^{-1} m=p$ and $a^{-1} b=j$. Note that $a^{-1} I_{2}$ is central invertible in $\&$ by Lemma 1 . Since $j \in J(R)$ and $j+(-$ $\varphi(p \otimes q))=: w \in j+\operatorname{Im} \varphi$, we have $\operatorname{det}\left(a^{-1} \alpha\right) \in J(R)$ and $\operatorname{det}\left(I_{2}-a^{-1} \alpha\right) \in J(R)$. This implies $a^{-1} \alpha$ is non-trivial strongly clean. Thus $x^{2}-\operatorname{tr}\left(a^{-1} \alpha\right)+\operatorname{det}\left(a^{-1} \alpha\right)=$ 0 is solvable by Theorem 9 , and so $a^{-1} \alpha$ is quasipolar, and so $\alpha$ is quasipolar by Lemma 8.

Case II. Let $\varphi(m \otimes n)=: u \in U(R)$. Now we prove that $t^{2}-t-w=0$ is solvable for all $w \in J(R)$. Let $w \in J(R)$ and write $\beta=\left[\begin{array}{cc}0 & u^{-1} m \\ n w & 1\end{array}\right]$. Since $\operatorname{det}(\beta)=0-$ $\varphi\left(u^{-1} m \otimes n w\right)=-u^{-1} w \varphi(m \otimes n)=-u^{-1} w u=-w \in J(R)$ and $\operatorname{det}\left(I_{2}-\beta\right)=$ $1-\operatorname{tr}(\beta)+\operatorname{det}(\beta)=-w \in J(R), \beta$ is a non-trivial strongly clean element, and so $t^{2}-t-w=0$ is solvable by Theorem 9. Hence $t^{2}-t+\frac{\operatorname{det}(\alpha)}{\operatorname{tr}(\alpha)^{2}}=0$ is solvable. This gives that $x^{2}-\operatorname{tr}(\alpha) x+\operatorname{det}(\alpha)=0$ is solvable. Consequently, $\alpha$ is quasipolar. We complete the proof.

Remark 7. According to the proof Theorem 11, we deduce that if 8 is strongly clean and $\operatorname{Im} \varphi \cap U(R) \neq \varnothing$, then $x^{2}-x-w=0$ is solvable for all $w \in J(R)$.

Theorem 12. Let $R$ be a commutative local ring with $\operatorname{Im} \varphi \subseteq J(R)$ and let $\alpha \in \mathcal{S}$. Then the following are equivalent.

(1) 8 is quasipolar.

(2) $x^{2}-(1+j) x+w=0$ is solvable for any $j \in J(R)$ and $w \in j+\operatorname{Im} \varphi$.

(3) $x^{2}-x+w=0$ is solvable for any $w \in \operatorname{Im} \varphi$.

Proof. (1) $\Rightarrow$ (2) Let $\alpha=\left[\begin{array}{cc}1 & -m \\ n & j\end{array}\right]$ where $j \in J(R), w=j+\varphi(m \otimes n)$. By (1), $\alpha$ is quasipolar. Since $\operatorname{det}(\alpha)=j+\varphi(m \otimes n) \in J(R)$ and $\operatorname{tr}(\alpha)=1+j \in U(R)$, we have that $x^{2}-(1+j) x+w=0$ is solvable by Theorem 10(2).

(2) $\Rightarrow(1)$ Let $\alpha=\left[\begin{array}{cc}a & m \\ n & b\end{array}\right] \in \delta$. If $a, b \in J(R)$ or $a, b \in U(R)$, then $\alpha \in J(\&)$ or $\alpha \in U(8)$, and so $\alpha$ is quasipolar. Hence, by Lemma 5 , we may assume that $a \in U(R)$ and $b \in J(R)$. Then we conclude that $a^{-1} \alpha=\left[\begin{array}{ll}1 & p \\ q & j\end{array}\right]$ where $a^{-1} n=q$, $a^{-1} m=p$ and $a^{-1} b=j$. Since $j \in J(R)$ and $j+(-\varphi(p \otimes q))=: w \in j+\operatorname{Im} \varphi$, by assumption, we get $x^{2}-(1+j) x+w=0$ is solvable. This gives that $a^{-1} \alpha$ is quasipolar, and so $\alpha$ is quasipolar by Lemma 8 . 
(1) $\Rightarrow$ (3) Similar to the proof of (1) $\Rightarrow$ (2).

(3) $\Rightarrow$ (1) Let $\alpha=\left[\begin{array}{cc}a & m_{1} \\ n_{1} & b\end{array}\right] \in$ \&. If $a, b \in J(R)$ or $a, b \in U(R)$, then $\alpha \in$ $J(8)$ or $\alpha \in U(8)$, and so $\alpha$ is quasipolar. Hence, by Lemma 5, we may assume that $a \in U(R)$ and $b \in J(R)$. Then we conclude that $a^{-1} \alpha=\left[\begin{array}{cc}1 & m_{2} \\ n_{2} & j\end{array}\right]=: \beta$ where $a^{-1} n_{1}=n_{2}, a^{-1} m_{1}=m_{2}$ and $a^{-1} b=j$. It is well known that $\beta$ is strongly clean if and only if $I_{2}-\beta$ is strongly clean. Hence, we now consider $I_{2}-\beta=$ $\left[\begin{array}{cc}0 & -m_{2} \\ -n_{2} & 1-j\end{array}\right]$. We directly see that $(1-j)^{-1}\left(I_{2}-\beta\right)=\left[\begin{array}{cc}0 & m \\ n & 1\end{array}\right]$ where $m=$ $-(1-j)^{-1} m_{2}$ and $n=-(1-j)^{-1} n_{2}$. By (3), we have that $x^{2}-x-\varphi(m \otimes n)=0$ is solvable, and so $(1-j)^{-1}\left(I_{2}-\beta\right)$ is (non-trivial) quasipolar. In view of Lemma 8, $I_{2}-\beta$ is (non-trivial) quasipolar; hence $\beta$ is (non-trivial) quasipolar by Remark 6 . Therefore, by Lemma 8, we deduce that $\alpha$ is quasipolar.

The following theorem is a generalization of Theorem 22 in [19].

Theorem 13. Let $R$ be a commutative local ring. If $\operatorname{Im} \varphi$ is a nilpotent ideal of $R$, then 8 is quasipolar.

Proof. We can assume that $(\operatorname{Im} \varphi)^{t}=0$ for some $t \in \mathbb{N}$. In this case, we also note that $\operatorname{Im} \varphi \subseteq J(R)$. By Theorem 12, it suffices to prove that $x^{2}-x+w=0$ is solvable for any $w \in \operatorname{Im} \varphi$. Let $w=\varphi(m \otimes n)$ and $f_{0}(x)=x^{2}-x+w=0$. We set

$$
W_{k}=\left\{w^{k} x^{2}-u x+w=0 \in R[x] \mid u \in 1+J(R), w \in J(R)\right\}
$$

for any $k \geq 0$. Then we have $f_{0}(w+w x)=w f_{1}(x)$ where $f_{1}(x)=w x^{2}+x+w \in$ $W_{1}$. Further, we see that $f_{1}(-w+w x)=w f_{2}(x)$ where $f_{2}(x)=w^{2} x^{2}-\left(2 w^{2}-\right.$ 1) $x+w^{2} \in W_{2}$. By iteration of this process, we get $f_{k-1}\left(w_{k}+w x\right)=w f_{k}(x)$ for all $k=1, \ldots, t$. Therefore,

$$
f_{t}\left(a_{t}\right) \in R
$$

$\Rightarrow f_{t-1}\left(a_{t-1}\right) \in w R$,

$\Rightarrow f_{t-2}\left(a_{t-2}\right) \in w^{2} R$

$\Rightarrow \quad f_{1}\left(a_{1}\right) \in w^{t-1} R$,

$\Rightarrow \quad f_{0}\left(a_{0}\right) \in w^{t} R=0$.

So we see that $x^{2}-x+w=0$ is solvable. We complete the proof.

We wind up the paper with some examples of special Morita contexts and quasipolar rings. In spite of the fact that it is difficult to find a pair of bimodule homomorphisms which satisfy the associativity rule, we supply the following examples.

Example 1. Let $\Gamma=[M, N, A, B, \psi, \phi]$ be a Morita context rings. Take $M=N=$ $R=A=B$ and $\psi=\phi=: \varphi$ is defined by $a \otimes b \mapsto s a b$ (or $a \otimes b \mapsto s^{2} a b$ ) where 
$s$ is central in $R$. Then $\delta$ is a generalized matrix ring over $R$ denoted by $K_{S}(R)$ (or $M_{2}\left(R ; s^{2}\right)$ ) (for more details see $[8,10,13,14,19,20]$ ). It is easy to see that $\delta$ is isomorphic to $M_{2}(R)$ when $s=1$. Therefore, we see that the special Morita context rings are a natural generalization of generalized matrix ring.

Example 2. Let $R$ be a local ring with $\operatorname{Im} \varphi=0$. Then $\delta$ is quasipolar if and only if $\delta$ is strongly clean and $M$ is cobleached (if and only if $M$ is uniquely bleached).

Proof. " $\Rightarrow$ " It is clear.

" $\Leftarrow$ " Assume that $\delta$ is strongly clean and $M$ is cobleached. Firstly, we prove that $M$ is bleached. Let $m \in M, u \in U(R)$ and $j \in J(R)$. Consider $\beta=\left[\begin{array}{cc}u & m \\ 0 & j\end{array}\right] \in \delta$. By assumption, $\beta$ is strongly clean. That is, there exists an idempotent $E$ such that $\beta-E$ is invertible and $E \in \operatorname{comm}(\beta)$. Take $E=\left[\begin{array}{ll}x & y \\ z & t\end{array}\right]$. Since $E^{2}=E$ and $\beta E=E \beta$, we directly see that $x^{2}=x, t^{2}=t, x u=u x, t j=j t$ and $u y+m t=$ $x m+y j$, and hence $x=0, t=1$ and $u y-y j=-m$ because $R$ is local and $\beta-E \in$ $U(8)$. Thus $M$ is uniquely bleached. Let $\alpha=\left[\begin{array}{cc}a & m \\ n & b\end{array}\right]$. It is enough to show that $\alpha$ is weakly quasipolar by Proposition 5. Without loss of generality, we may assume that $a \in U(R)$ and $b \in J(R)$. As $M$ is bleached, there exist $x, y \in M$ such that $u x-x j=-m$ and $j y-y u=n$. Write $E=\left[\begin{array}{ll}0 & x \\ y & 1\end{array}\right]$. This gives that $E^{2}=E$, $E \in \operatorname{comm}(\alpha)$ and $\alpha E \in J(\S)$, and so $\alpha$ is weakly quasipolar.

Example 3. We consider the special Morita context $\delta=\left[\begin{array}{cc}R & M \\ M & R\end{array}\right]$ where $R=$ $\mathbb{Z}_{4}, M=\mathbb{Z}_{2} \oplus \mathbb{Z}_{2}$ and $\varphi: M \otimes M \rightarrow \mathbb{Z}_{4}$ is defined by $\varphi((\bar{a}, \bar{b}) \otimes(\bar{c}, \bar{d}))=\overline{2(a c+b d)}$. This implies that $\varphi$ satisfy the associativity conditions and $\operatorname{Im} \varphi=\{\overline{0}, \overline{4}\}$, and $\operatorname{so} \operatorname{Im} \varphi$ is nilpotent ideal in $R$. Hence, by Theorem $13,8=[R, M, \varphi]$ is quasipolar.

\section{REFERENCES}

[1] G. Azumaya, "Strongly $\pi$-regular rings," J. Fac. Sci. Hokkaido Univ. Ser. I., vol. 13, pp. 34-39, 1954.

[2] J. Cui and J. Chen, "When is a $2 \times 2$ matrix ring over a commutative local ring quasipolar?" Comm. Algebra, vol. 39, no. 9, pp. 3212-3221, 2011, doi: 10.1080/00927872.2010.499118.

[3] J. Cui and J. Chen, "Notes on quasipolar rings," J. Math. Study, vol. 45, no. 2, pp. 167-174, 2012.

[4] J. Cui and J. Chen, "Quasipolar triangular matrix rings over local rings," Comm. Algebra, vol. 40, no. 2, pp. 784-794, 2012, doi: 10.1080/00927872.2010.538457.

[5] M. P. Drazin, "Pseudo-inverses in associative rings and semigroups," Amer. Math. Monthly, vol. 65, pp. 506-514, 1958, doi: 10.2307/2308576.

[6] O. Gürgün, "On cline's formula for some certain elements in a ring," An. Ştiinţ. Univ. Al. I. Cuza Iassi. Mat. (N.S.), vol. 41, no. 1, pp. 15-26, 2016.

[7] O. Gürgün, "Extensions of quasipolar rings," Turk. J. Math., vol. 1, no. 2, pp. 403-410, 2017. 
[8] O. Gürgün, S. Halicioglu, and A. Harmanci, "Strong j-cleanness of formal matrix rings," Adv. Stud. Contemp. Math. (Kyungshang), vol. 24, no. 4, pp. 483-498, 2014.

[9] R. Harte, "On quasinilpotents in rings," Panamer. Math. J., vol. 1, pp. 10-16, 1991.

[10] Q. Huang, G. Tang, and Y. Zhou, "Quasipolar property of generalized matrix rings," Comm. Algebra, vol. 42, no. 9, pp. 3883-3894, 2014, doi: 10.1080/00927872.2013.796964.

[11] J. J. Koliha, "A generalized Drazin inverse," Glasgow Math. J., vol. 38, no. 3, pp. 367-381, 1996, doi: 10.1017/S0017089500031803.

[12] J. J. Koliha and P. Patricio, "Elements of rings with equal spectral idempotents," J. Aust. Math. Soc., vol. 72, no. 1, pp. 137-152, 2002, doi: 10.1017/S1446788700003657.

[13] P. A. Krylov, "On the isomorphism of generalized matrix rings," Algebra Logika, vol. 47, no. 4, pp. 456-463, 524, 2008, doi: 10.1007/s10469-008-9016-y.

[14] P. A. Krylov and A. A. Tuganbaev, "Modules over formal matrix rings," Fundam. Prikl. Mat., vol. 15, no. 8, pp. 145-211, 2009, doi: 10.1007/s10958-010-0133-5.

[15] Y. Liao, J. Chen, and J. Cui, "Cline's formula for the generalized Drazin inverse," Bull. Malays. Math. Sci. Soc. (2), vol. 37, no. 1, pp. 37-42, 2014.

[16] W. K. Nicholson, "Strongly clean rings and Fitting's lemma," Comm. Algebra, vol. 27, no. 8, pp. 3583-3592, 1999, doi: 10.1080/00927879908826649.

[17] A. D. Sands, "Radicals and Morita contexts," J. Algebra, vol. 24, pp. 335-345, 1973, doi: 10.1016/0021-8693(73)90143-9.

[18] G. Song and X. Guo, "Diagonability of idempotent matrices over noncommutative rings," Linear Algebra Appl., vol. 297, no. 1-3, pp. 1-7, 1999, doi: 10.1016/S0024-3795(99)00059-2.

[19] G. Tang and Y. Zhou, "Strong cleanness of generalized matrix rings over a local ring," Linear Algebra Appl., vol. 437, no. 10, pp. 2546-2559, 2012, doi: 10.1016/j.laa.2012.06.035.

[20] G. Tang and Y. Zhou, "A class of formal matrix rings," Linear Algebra Appl., vol. 438, no. 12, pp. 4672-4688, 2013, doi: 10.1016/j.laa.2013.02.019.

[21] Z. Wang and J. Chen, "Pseudo Drazin inverses in associative rings and Banach algebras," Linear Algebra Appl., vol. 437, no. 6, pp. 1332-1345, 2012, doi: 10.1016/j.laa.2012.04.039.

[22] Z. Ying and J. Chen, "On quasipolar rings," Algebra Colloq., vol. 19, no. 4, pp. 683-692, 2012, doi: 10.1142/S1005386712000557.

Authors' addresses

O. Gürgün

Ankara University, Department of Mathematics, 06100 Ankara, Turkey

E-mail address: orhangurgun@gmail.com

S. Halicioglu

Ankara University, Department of Mathematics, 06100 Ankara, Turkey

E-mail address: halicilankara.edu.tr

\section{A. Harmanci}

Hacettepe University, Department of Mathematics, 06800 Ankara, Turkey

E-mail address: harmanci@hacettepe.edu.tr 